\title{
Suppression of food allergy by maternal antigen-specific IgG in murine model
}

\author{
Hirotaka Yamashita, Kenichi Saneyasu, Hiroyuki Tanaka, Naoki Inagaki
}

\section{Laboratory of Pharmacology, Gifu Pharmaceutical University, Japan}

A number of patients with food allergy is increasing. Anaphylaxis caused by food has occurred in early time in the life. The fact could suggest that development of food allergy was related with parental immunity. Then, we confirmed effect of parental food allergy on development of food allergy to use our murine food allergy model. Previously, we established models for food allergy and oral tolerance to use ovalbumin (OVA) as food antigen. BALB/c mice were sensitized by administration of OVA/alum intraperitoneally. The sensitized mice were treated with OVA orally several times. Food allergy was estimated by drop of body temperature, fecal water content, and serum OVA-specific IgE and IgG1 levels. In the oral tolerance model, OVA was treated orally before the sensitization with OVA/alum. The elevations of OVA-specific $\mathrm{IgE}$ and anaphylaxis for OVA were suppressed by the induction of oral tolerance. Next, female and male mice with food allergy or oral tolerance were mated with each other to investigate effects of parental immune condition on infant food allergy. As results, OVA-specific IgE did not increase and food allergy could not be induced in offspring from pairs of mice with food allergy. However, we could detect a small amount of OVA-specific IgG1 in blood of the na\&\#239; ve infant mice born from parents with food allergy. We speculated that OVA-specific IgG derived from the mother prevented food allergy. Then, food allergy-serum containing OVA-specific IgG, monoclonal OVA-specific IgG1, monoclonal OVAspecific $\operatorname{IgG2a}$ and a mixture of monoclonal IgG1 and monoclonal IgG2a were injected into na\&\#239; ve mice, and the mice were sensitized by OVA/alum. As results, increase in OVA-specific IgE in the mice injected food allergy-serum was significantly ameliorated, but not monoclonal IgGs. Additionally, we detected OVA-IgG immune complex in food allergymother's serum. Our data might indicate that maternal specific IgG conjugated food antigen is an important factor related to the development of food allergy and acquiring tolerance. 